\title{
Gas sensing using terahertz time-domain spectroscopy
}

\author{
D.M. Mittleman ${ }^{1}$, R.H. Jacobsen ${ }^{2}$, R. Neelamani ${ }^{1}$, R.G. Baraniuk ${ }^{1}$, M.C. Nuss ${ }^{3}$ \\ ${ }^{1}$ Electrical and Computer Engineering Dept., MS-366, 6100 Main Street, Rice University, Houston, TX 77005, USA \\ (Fax: +1-713/524-5237, E-mail: daniel@ rice.edu) \\ ${ }^{2}$ Tele Danmark Udvikling, UPTA 11-190, Sletvej 30, 8310 Tranbjerg J, Denmark \\ ${ }^{3}$ Bell Laboratories-Lucent Technologies, Room 4B-429, 101 Crawfords Corner Road, Holmdel, NJ 07733, USA
}

Received: 25 February 1998

\begin{abstract}
A method for detection and identification of polar gases and gas mixtures based on the technique of terahertz time-domain spectroscopy is presented. This relatively new technology promises to be the first portable far-infrared spectrometer, providing a means for real-time spectroscopic measurements over a broad bandwidth up to several THz. The measured time-domain waveforms can be efficiently parameterized using standard tools from signal processing, including procedures developed for speech recognition applications. These are generally more efficient than conventional methods based on Fourier analysis, and are easier to implement in a real-time sensing system. Preliminary results of real-time gas mixture analysis using a linear predictive coding algorithm are presented. A number of possible avenues for improved signal processing schemes are discussed. In particular, the utility of a wavelet-based signal analysis for tasks such as denoising is demonstrated.
\end{abstract}

Spectroscopic methods for the sensing and identification of gases have shown great promise, owing to their inherent non-invasive nature, relative simplicity, and high selectivity. The vast majority of the work in this area has relied on the "fingerprint" absorption in the mid-infrared $(\lambda \approx 2-20 \mu \mathrm{m})$, where molecular vibrations often provide a unique signature. Both incoherent (for example, Fourier-transform infrared, FTIR) [1] and laser-based coherent sources [2] have been commonly employed. These have demonstrated sensitive detection of many gases including greenhouse gases such as $\mathrm{CO}, \mathrm{CO}_{2}$, and $\mathrm{CH}_{4}$, chemical etchants such as $\mathrm{HCl}$ and $\mathrm{HF}$, and common smokestack pollutants such as $\mathrm{SO}_{2}$ and $\mathrm{N}_{2} \mathrm{O}$. Despite these promising results, significant challenges remain, particularly in expanding the range of gases amenable to spectroscopic detection.

In contrast to mid-infrared gas sensing, the use of farinfrared or terahertz $\left(1 \mathrm{THz}=10^{12} \mathrm{~Hz}\right)$ radiation for sensing purposes is a field in relative infancy. In this frequency range, from $\lambda \approx 3 \mathrm{~mm}$ to $50 \mu \mathrm{m}$ (corresponding to frequencies between 0.1 and $6 \mathrm{THz}$ ), many polar molecules exhibit unique spectral signatures arising from transitions between rotational quantum levels. The use of these absorption signatures for detection or identification of gases is very much complementary to the more well-established mid-IR techniques, and will greatly expand the number of gas species that can be detected via laser-based methods. Yet, the development of THz sensing tools has been hindered in large part due to the lack of suitable radiation sources and detectors for use in the farinfrared.

Within the last decade, a number of new approaches to the generation and detection of $\mathrm{THz}$ radiation have been pursued with increasing interest. These techniques, based on frequency conversion using nonlinear optics [3-8], are often simpler, more reliable, and potentially much less expensive than the more traditional approaches such as molecular vapor lasers, free electron lasers, and synchrotrons. Although many of these nonlinear optical techniques were pioneered in the late 1960s or early 1970s [9], it is only relatively recently that it has become plausible to consider "real-world" applications based on these $\mathrm{THz}$ technologies. This excitement has been spurred in part by a number of important advances. First, a range of new fabrication techniques have been developed for nonlinear optical materials, such as the low-temperature growth of semiconductors and periodic poling. Second, there have been substantial improvements in the reliability, stability, size, and cost of the lasers required for these devices. The advances in the ultrafast pulsed laser systems are particularly notable, with the cumbersome argon-ion-pumped Kerr-lensmode-locked Ti:sapphire lasers giving way to more compact and less costly all-solid-state systems [10] or to mode-locked fiber lasers [11].

In this paper, we present a review of recent results in automated real-time gas sensing involving one of the more promising of these new $\mathrm{THz}$ techniques, known as terahertz time-domain spectroscopy, or THz-TDS. The THz-TDS system relies on the use of femtosecond laser pulses for the generation and detection of $\mathrm{THz}$ radiation, and has been a beneficiary of the rapid progress in this enabling technology. Using THz-TDS, one can obtain a rapid spectral measurement over a very large bandwidth in the terahertz range, where many gases exhibit "fingerprint" absorption spectra 
arising from rotational quantum transitions, analogous to the familiar vibrational fingerprint region. These rotational line spectra can be used for gas identification and gas mixture analysis. Broadly speaking, the advantages of this technique are straightforward: relatively easy access to a previously unexploited spectral range will expand the number of gas species amenable to remote detection. Many gases may be detected more efficiently or accurately by relying on their relatively simple rotational spectra, rather than on their more complex vibrational or rovibrational signatures. Other advantages include the great flexibility afforded by the extremely broad bandwidth accessible with THz-TDS, and the coherent detection, which permits far-infrared spectroscopic measurements of plasmas, flames, and other challenging samples.

Here, we describe the THz-TDS technique, highlighting those aspects which are uniquely advantageous for the purposes of gas sensing. We focus particularly on how the nature of the measured signals lends itself to rapid and highly selective data analysis. We review recent results involving the implementation of a real-time gas sensing system, with emphasis on the adaptation of powerful signal processing protocols for gas and gas mixture analysis. Because the ultimate sensitivity limits for a given gas will depend largely on the effectiveness of the signal processing, we have concentrated our recent efforts on further development of these algorithms. We provide a brief discussion of the future directions of this research, noting in particular the efficacy of wavelet analyses of the THz-TDS signals for signal processing challenges such as deconvolution and denoising.

\section{Terahertz time-domain spectroscopy}

The THz-TDS system described here has been reviewed extensively in the recent literature [12-14]. With THz-TDS, one overcomes many of the difficulties traditionally associated with research in the far-infrared, such as the need for cryogenics for cooling detectors. Because of this, a great deal of interest has been generated by the potential for novel farinfrared spectroscopic measurements [15-18], and by the recent demonstrations of imaging using $\mathrm{THz}$ waves [19-21]. The necessity for a source of fs pulses has hindered the development of this $\mathrm{THz}$ source for applications such as gas sensing, but the recent progress mentioned above has minimized many of these concerns. With the fs laser system no longer a substantial impediment to commercialization, the development of a compact portable coherent terahertz spectrometer is currently under way.

A typical THz-TDS gas sensing setup is shown in Fig. 1. Pioneered in the late 1980s by groups at Bell Laboratories [3] and IBM's T.J. Watson Research Center [23], this system relies on an ultrafast laser system, producing a train of optical (usually near-infrared) pulses, of $\approx 100-\mathrm{fs}\left(10^{-13} \mathrm{~s}\right) \mathrm{du}-$ ration. This pulse train is split into two arms, one for the $\mathrm{THz}$ emitter and one for the detector. The emitter is usually a direct-gap semiconductor (typically GaAs) with some simple antenna structure lithographically defined on its surface. This high-impedance dipole emitter is dc-biased, typically with a few tens of volts. When a fs pulse excites the semiconducting material in the antenna gap with an aboveband-gap excitation, the photogenerated charge carriers are accelerated by the applied bias field. The rise time of this pho-

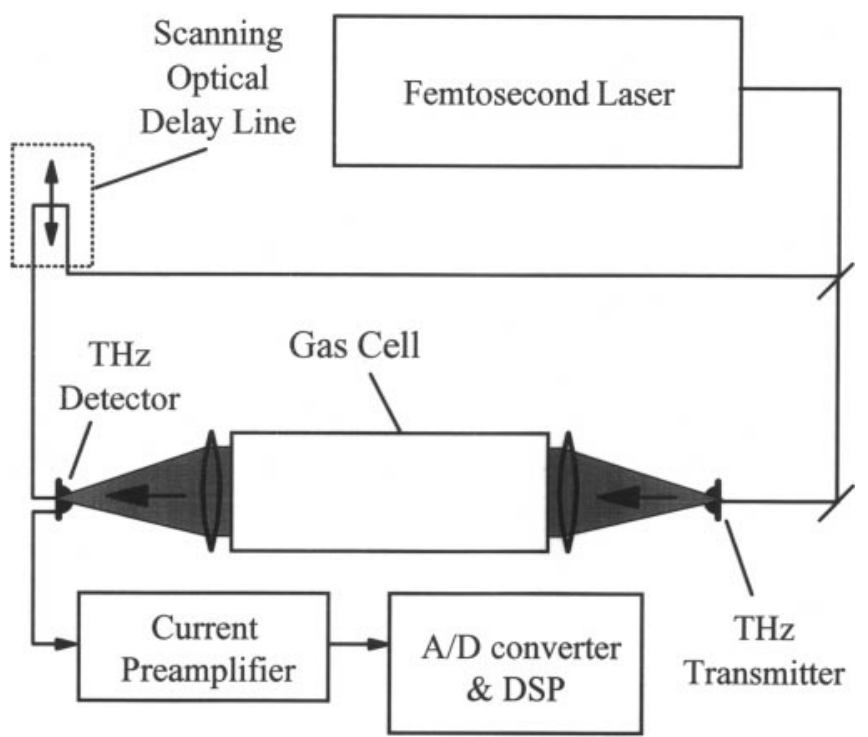

Fig. 1. Experimental setup for gas sensing measurements based on terahertz time-domain spectroscopy

tocurrent can be nearly as fast as the fs optical pulse duration. This transient current radiates according to Maxwell's equations, $E(t) \propto \partial j(t) / \partial t$. Because $j(t)$ is approximately a step function, this radiated field is close to a single-cycle pulse, with extremely large fractional bandwidth. The radiated field can also contain contributions from effects other than charge transport, such as optical rectification [4], although in the experiments described here this contribution is negligible.

When the radiation emerges from the rear surface of the emitter, it is collected, first with a substrate lens, then using conventional far-infrared optics. The resulting $\mathrm{THz}$ beam can be collimated and focused just as any laser beam, except that the long-wavelength radiation diffracts more readily than optical beams [23]. Even so, a well-collimated $\mathrm{THz}$ beam of $\approx 1-\mathrm{cm}$ radius can propagate for several meters before diffraction becomes significant. This large beam diameter precludes the use of most commercially available multi-pass cells, such as those used in many mid-infrared gas sensing systems to increase optical path lengths. One could probably design a multi-pass arrangement appropriate for the $\mathrm{THz}$ beam, but for present purposes a single-pass cell of length $\approx 30 \mathrm{~cm}$ has been used (Fig. 1).

The detection of these $\mathrm{THz}$ transients is accomplished using the second arm of the fs beam. The traditional scheme relies on photoconductive sampling in an antenna similar to that used in the emission process. In this case, the $\mathrm{THz}$ pulse provides the bias field, which drives the photogenerated carriers in the antenna circuit. This photocurrent is sampled by varying the delay of the fs gate beam with respect to the $\mathrm{THz}$ beam, using an optical delay line. This measurement relies on the fact that the photogenerated carriers have a high mobility (because the induced current depends on the carrier mobility) and also a fast carrier lifetime (because a sampling measurement is only useful if the sampling window is fast compared to the transients being measured). These requirements place rather stringent demands on the semiconductor materials used in the detector. Currently, low-temperaturegrown (LT) GaAs and radiation-damaged silicon-on-sapphire are the two most common choices. These materials have been 
used for spectroscopic measurements spanning bandwidths from below $0.1 \mathrm{THz}$ to beyond $5 \mathrm{THz}$.

A typical $\mathrm{THz}$ pulse measured in this fashion is shown in Fig. 2. Here, curve (a) shows the measured photocurrent as a function of the delay between the two beams. This photocurrent is essentially proportional to the terahertz electric field, $E_{\mathrm{THz}}(t)$. On the bottom (curve (b)), the magnitude of the Fourier transform of $E_{\mathrm{THz}}(t)$ is shown. In this data, the antenna used for detection provided useful bandwidth from $100 \mathrm{GHz}$ to $\approx 2.5 \mathrm{THz}$. The lower limit is determined by diffraction losses in the optical system, as well as the acceptance bandwidth of the receiver antenna. The upper limit is largely determined by the free-carrier lifetime in the semiconductor, which limits the minimum temporal width of the sampling gate. In principle, the bandwidth is also limited by the duration of the fs pulses used to gate the antenna, but the free-carrier lifetime is, in general, longer than the pulse duration and is therefore the more important limiting factor [24].

The speed at which these waveforms can be measured depends on several factors, including the speed of the A/D conversion, the required signal-to-noise ratio of the measurement, and the speed of the mechanical optical delay line. Using a scanning optical delay line (a retroreflector mounted on a galvanometric motor), these $\mathrm{THz}$ waveforms can be measured in only a few tens of milliseconds with signal-
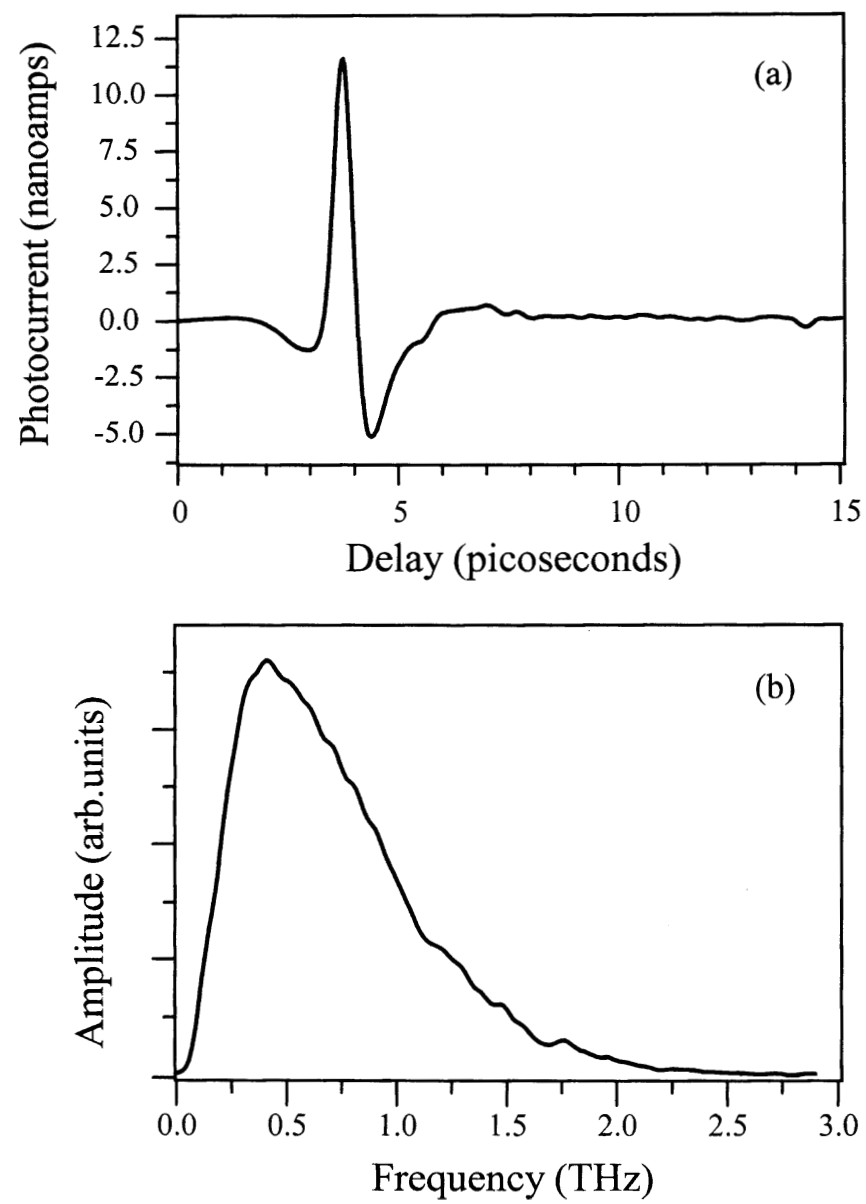

Fig. 2a,b. THz waveform measured using photoconductive sampling. a Average photocurrent (proportional to $\mathrm{THz}$ electric field) as a function of optical delay. $\mathbf{b}$ amplitude of Fourier transform of the signal in $\mathbf{a}$, showing the spectral content of the radiation to-noise in excess of $10^{3}$. The scanning delay line can be driven by a triangle wave, providing a linear variation of optical delay with time, at a rate of $10-100 \mathrm{~Hz}$. This effectively down-converts the $\mathrm{THz}$ waveforms into the $\mathrm{kHz}$ range, where they can be processed with slow electronics. This fast data acquisition permits rapid analysis of the full spectral bandwidth spanned by the $\mathrm{THz}$ pulse, and thus real-time sensing and identification of gases with absorption signatures within the measured bandwidth. Further, because the measured waveforms are in the $\mathrm{kHz}$ range, they are well suited for processing with available digital signal processing algorithms. These algorithms are often more efficient than the more familiar frequency-domain analogs, and can provide a high degree of selectivity in gas identification. An example of an implementation of such a processing scheme is described below.

Recently, a second detection scheme has been gaining in popularity. This newer technique is known as freespace electro-optic sampling [25]. Here, the THz beam copropagates with a synchronized fs probe pulse in an electrooptically active material. The electric field of the $\mathrm{THz}$ pulse induces a polarization rotation of the fs probe beam, via the Pockel's effect. This rotation can be measured as a function of the delay between the $\mathrm{THz}$ pulse and the optical pulse, giving a direct measurement of the $\mathrm{THz}$ electric field. In contrast with the photoconductive technique outlined above, the electro-optic nonlinearity is nonresonant, and thus responds essentially instantaneously to the applied field. As a result, this technique can provide much more detection bandwidth, limited by the duration of the fs probe pulse. Using ZnTe as a detector, bandwidths as large as $37 \mathrm{THz}$ have been reported [26]. Presently, the photoconductive sampling technique is more favorable for gas sensing applications, because of the ease of implementing real-time measurements. A fast scanning delay line can be used to acquire the $\mathrm{THz}$ waveforms with the electro-optic sampling technique, but the hardware requirements are somewhat more cumbersome [27]. Also, the use of fiber-optic-coupled detection antennas is substantially easier with the photoconductive technique, resulting in a system which is much less sensitive to alignment and external perturbation. In situations where waveform acquisition times of a few minutes are tolerable, the broader bandwidth available from electro-optic sampling will be quite valuable.

It should be emphasized that both of these detection techniques are coherent; they measure the $\mathrm{THz}$ electric field $E_{\mathrm{THz}}(t)$, not the intensity $\left|E_{\mathrm{THz}}(t)\right|^{2}$. As a result, the phase information is preserved, and one may determine both the real and imaginary parts of a sample's dielectric function simultaneously, without resorting to Kramers-Kronig analysis. This phase-sensitive detection has a further advantage in the near-complete rejection of incoherent radiation. Thus, these detectors generally operate at room temperature and in ambient light. This obviates the need for the cumbersome cryogenic apparatus typically required in far-infrared systems. Additionally, it permits spectroscopic and imaging studies of hot samples such as flames and plasmas, whose large thermal signatures would ordinarily swamp an incoherent detector [28]. An example of this is shown in Fig. 3, which is an image of $\mathrm{a} \approx 1-\mathrm{cm}$ gas flame [14]. The waveforms collected at each pixel of this image display no measurable variation in spectral amplitude. Instead, small changes in phase are detected, corresponding to variations in the transit time of the $\mathrm{THz}$ pulse through different regions of the flame. This 


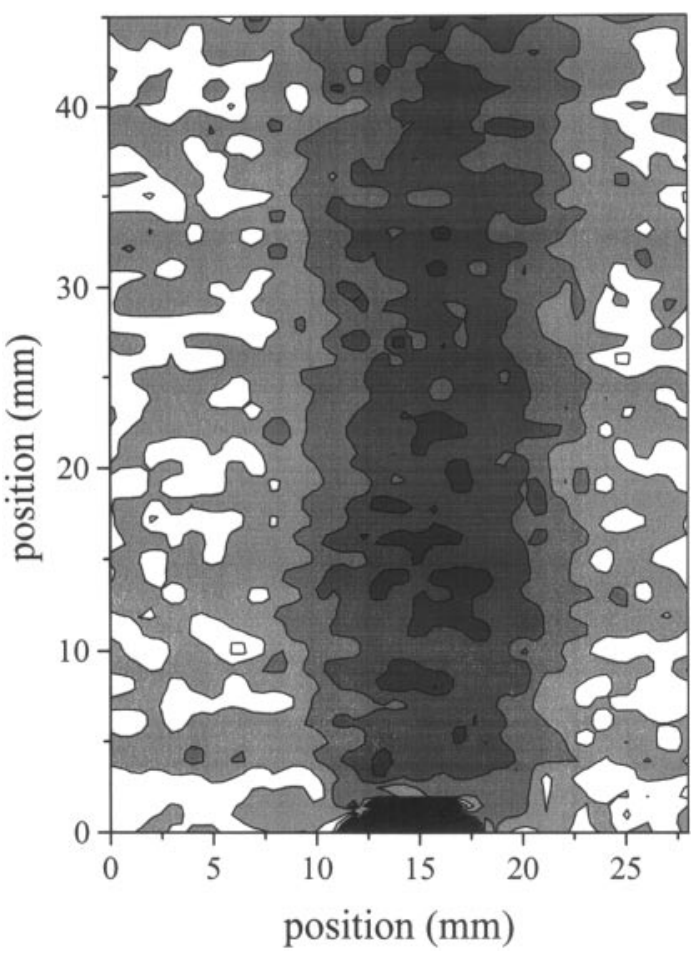

Fig. 3. THz image of a small gas flame, in which each contour corresponds to a shift in the transit time of the THz pulse of $5 \mathrm{fs}$. The pulse travels faster through the center of the flame because of the lower density (and thus lower index) of heated air

variation is due to the change in refractive index of the air which results from heating, and is displayed as gray scale contours. Adjacent contours correspond to shifts in the arrival time of only $5 \mathrm{fs}$, corresponding to changes in the phase of $\approx 0.015$ radians. The ability to perform measurements such as this opens up many new possibilities in gas sensing, including the monitoring of gases in ovens or of plasmas, for example.

\section{Gas sensing with terahertz time-domain spectroscopy}

Although far-infrared spectroscopy of gases has been a vibrant area of research for many years [29], the use of active devices in the $\mathrm{THz}$ range for gas sensing and identification is only just beginning to be explored [30,31]. The more mature technology based on mid-IR spectral signatures has demonstrated sensitive detection of many gases at the level of a few parts per billion, based on vibrational fingerprint spectroscopy [2]. However, larger molecules such as polar organic solvents and chlorofluorocarbons tend to exhibit extremely complex and congested line shapes in this spectral range. As a result, distinguishing an individual molecular species based purely on mid-infrared spectroscopy can be quite challenging. In the far-infrared regime, the line shapes tend to simplify considerably, since one is left with pure rotational spectra. In many cases, these spectra can be characterized by a small number of rotational energy parameters. Thus, characterization of more complex molecules is frequently easier with pure rotational spectra than it is when vibrational levels are also excited. A device that spans the rotational spectral region constitutes a powerful complement to established sens- ing technologies, expanding the number of gases amenable to spectroscopic identification. Here, the gas sensing methods based on THz-TDS are described in some detail, with particular focus on the important signal processing issues. The examples presented here are chosen, not because they represent insurmountable challenges with more well established technologies, but because they serve to illustrate the capabilities and limitations of the time-domain spectrometer.

In recent years, THz-TDS has been used as a tool for spectroscopic studies of a number of gases, including water vapor [32], methyl chloride [33], and nitrous oxide [34]. As with most polar molecules, these gases have characteristic fingerprint absorption spectra within the bandwidth of the farinfrared radiation. These spectra, which originate from transitions between rotational quantum levels, can be theoretically modeled quite accurately [35]. The narrow rotational absorption lines attenuate selected frequency components within the bandwidth of the $\mathrm{THz}$ beam as it propagates through the sample under study. The resulting waveform, measured in the time domain, exhibits small oscillatory features following the main pulse. Examples of this are depicted in Figs. 4 and 5, which show the waveforms that result when the THz pulse is passed through water vapor and acetonitrile (methyl cyanide, $\mathrm{CH}_{3} \mathrm{CN}$ ) vapor, respectively. In the case of water (Fig. 4) the irregularly spaced absorption lines produce a complex beat pattern following the initial pulse. These absorption lines are observed in the Fourier transform of the $\mathrm{THz}$ waveform as
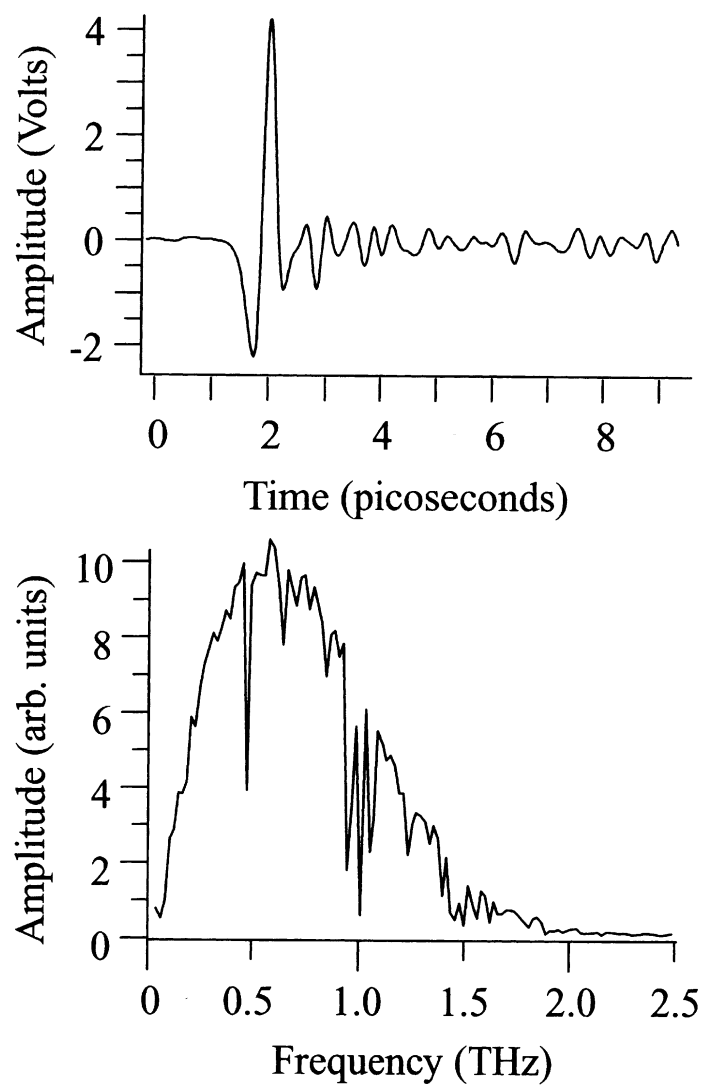

Fig. 4. THz waveform modified by propagation through humid air. The oscillations following the initial pulse result from the effects of water vapor. The $\mathrm{H}_{2} \mathrm{O}$ absorption lines attenuate selected narrow frequency intervals within the spectrum of the $\mathrm{THz}$ pulse, as seen in the Fourier transform, at right 

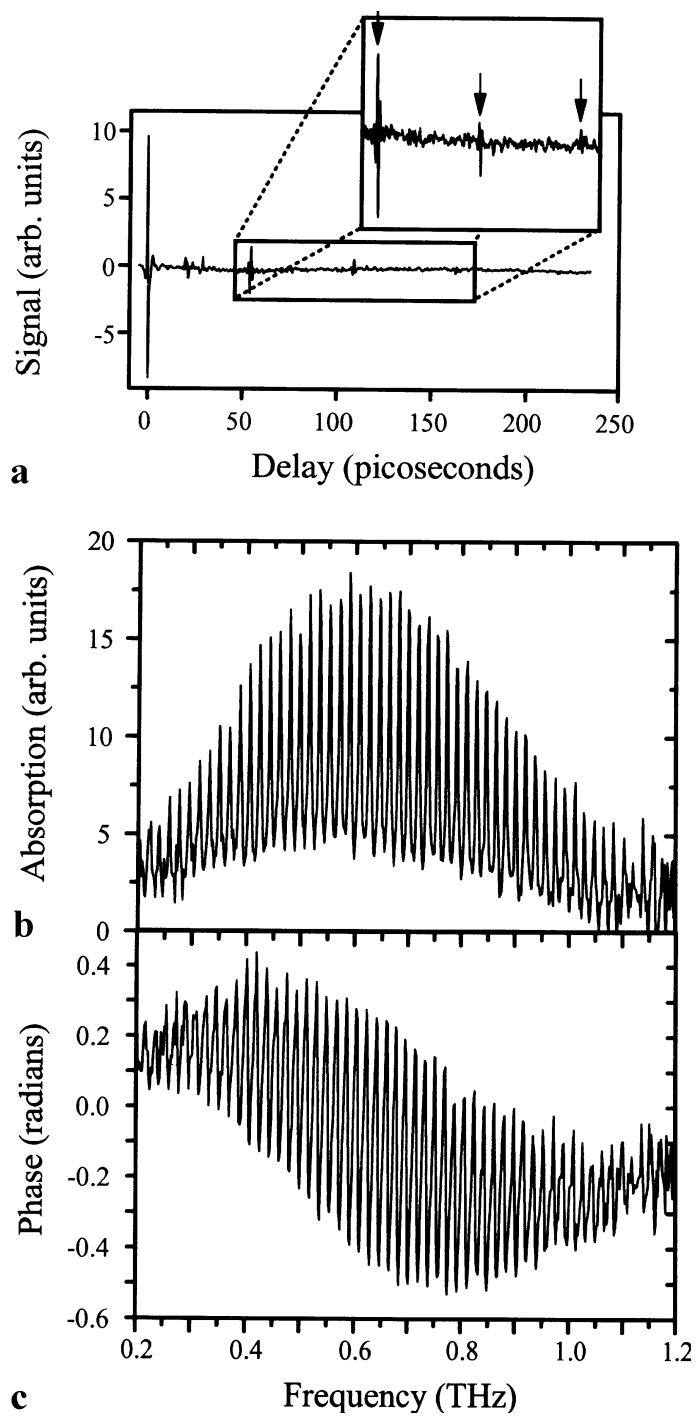

Fig. 5. a THz waveform modified by propagation through acetonitrile vapor, and the corresponding absorption (b) and dispersion (c) spectra. Arrows in $\mathbf{a}$ indicate the positions of the periodic echoes which result from the coherent excitation of the rotational manifold

sharp dips in the spectrum. In the case of the symmetric rotor acetonitrile, the regularly spaced rotational manifold generates a series of recurrences or echoes of the initial pulse [36], at temporal delays that are multiples of the inverse of the rotational line spacing (Fig. 5). In some cases, more than 25 such echoes can be observed, extending for more than $1 \mathrm{~ns}$ after the initial transient [33]. The absorption coefficient and dispersive phase can be independently derived from this timedomain data by Fourier transform, and are shown in Fig. 5b,c.

It should be noted that the linewidths of these rotational transitions are generally far narrower than the spectral resolution of the THz-TDS measurements, which is determined by the range of the temporal scan and is typically 1-10 GHz. A method has been reported for high-resolution (better than $1 \mathrm{MHz}$ ) gas phase spectroscopy using demodulation of a fs $\mathrm{THz}$ source, but the advantage of coherent detection is lost [37]. The coherent detection methods described above, although not well suited for detailed lineshape measurements due to the lack of sub- $\mathrm{MHz}$ resolution, are more than sufficient for distinguishing between different gas species in most cases, and are therefore adequate for many practical gas sensing applications.

Indeed, a great deal of information can be gained directly from the time-domain waveform, without the need for a Fourier transform. For example, in Fig. 5a, the observed delay $\Delta t$ between the periodic recurrences in the time-domain waveform (indicated by arrows) is roughly $54 \mathrm{ps.} \mathrm{Since} \mathrm{the}$ rotational line spacing is given, to a good approximation by $\Delta v=2 B(J+1)$, it follows that $B=(2 \Delta t)^{-1} \approx 9.2 \mathrm{GHz}$ for acetonitrile, in good agreement with the literature value [38]. Values for the rotational constant derived in this fashion can be used to provide a rapid identification of the absorbing species. Given the nature of the THz-TDS signals, such timedomain methods for characterizing and identifying molecular species are more natural than frequency-domain techniques, and often more efficient as well.

The selectivity of this technique is demonstrated in Fig. 6, which shows the temporal waveforms obtained by passing the $\mathrm{THz}$ beam through a sample of acetonitrile $\left(\mathrm{CH}_{3} \mathrm{CN}\right.$, curve a) and its fully deuterated analog $\left(\mathrm{CD}_{3} \mathrm{CN}\right.$, curve b). Only the first $\approx 70 \mathrm{ps}$ of each waveform is shown, so only the first recurrence is visible in each case. The difference between the two gases is quite dramatic, as the temporal delay of the first echo shifts from $\Delta t \approx 54 \mathrm{ps}$ in $\mathrm{CH}_{3} \mathrm{CN}$ to $64 \mathrm{ps}$ in $\mathrm{CD}_{3} \mathrm{CN}$. This reflects the decrease in the rotational constant of the molecule from $\approx 9.2 \mathrm{GHz}$ to $\approx 7.8 \mathrm{GHz}$, resulting from the larger moment of inertia of the deuterated molecule [38]. As mentioned above (see Fig. 3), extremely small shifts in $\Delta t$ (less than $10 \mathrm{fs}$ ) can be detected using THz-TDS. This is therefore a sensitive method for distinguishing among several closely related gas species.

Additionally, the use of $\mathrm{THz}$ spectroscopy for gas identification is greatly facilitated by the simplicity of the spectral signatures of these gases. Because of the periodicity of the rotational manifold, the $\mathrm{THz}$ spectra (and consequently the measured time-domain waveforms) can be parameterized by a single number. Although some information is neglected (for example, the envelope of the rotational manifold), that which remains (for example, the value of the rotational constant $B$ ) is often sufficient for gas identification. This is in contrast

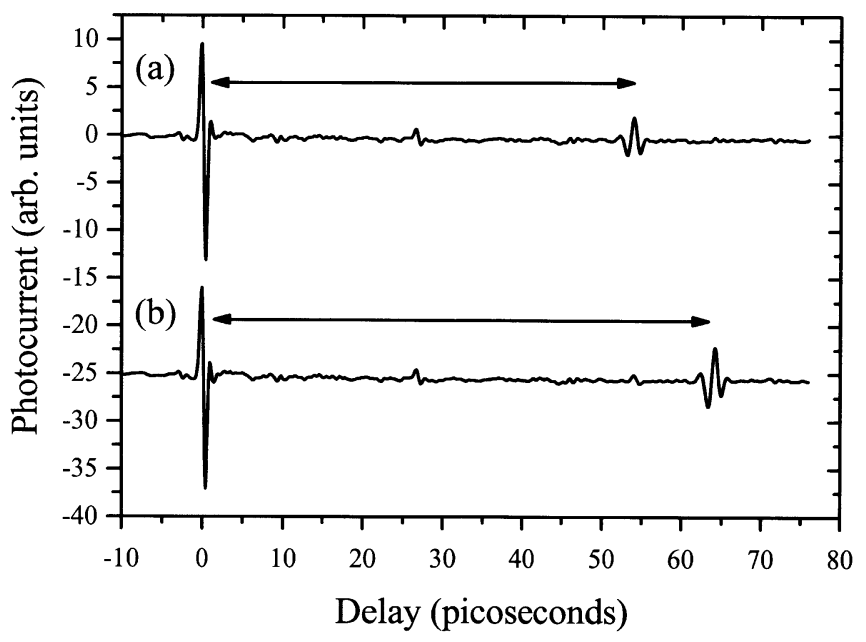

Fig. 6. Time-domain waveforms showing the effects of propagation through small amounts of gaseous $\mathrm{CH}_{3} \mathrm{CN}$ (a) and $\mathrm{CD}_{3} \mathrm{CN}$ (b). The shift in the delay of the first echo (indicated by arrows) results from the larger moment of inertia of the deuterated species. Waveforms offset for clarity 


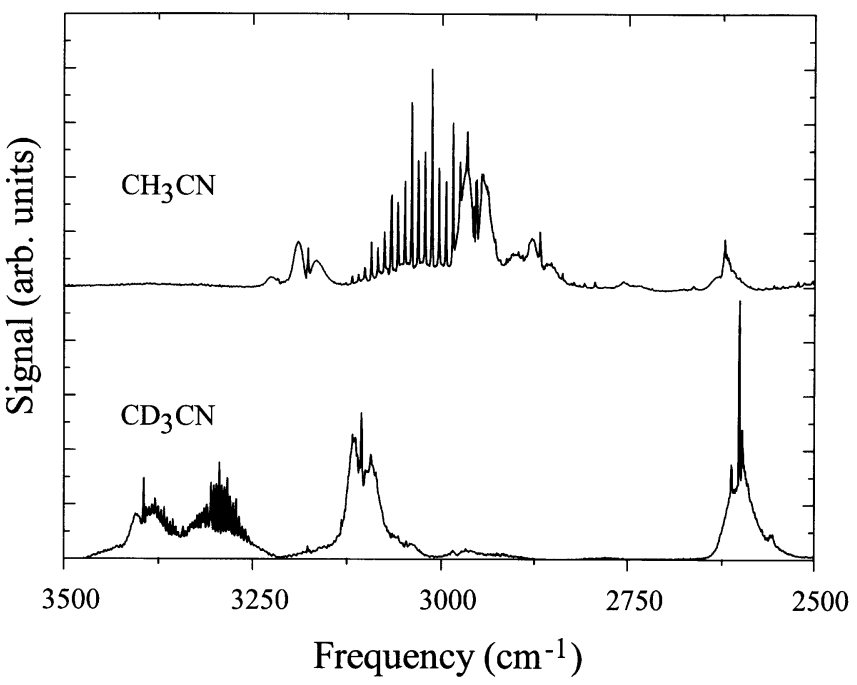

Fig. 7. Mid-infrared absorption spectra of $\mathrm{CH}_{3} \mathrm{CN}$ and $\mathrm{CD}_{3} \mathrm{CN}$, measured with a commercial FTIR spectrometer. Spectra vertically offset for clarity

with many examples in sensing based on mid-infrared vibrational spectroscopy, where even the spectra of relatively simple molecules such as acetonitrile are quite complicated [39] and require detailed analysis for band assignment. Figure 7 illustrates this point, showing the mid-infrared spectra of the two gases of Fig. 6, obtained using a commercial FTIR spectrometer. These two spectra, although quite different from one another, are both rather complex, as a result of the interplay between different vibrational and rovibrational modes of the molecules. Evidently, distinguishing between these two species is far simpler and more amenable to automated analysis with the $\mathrm{THz}$ time-domain signals. One can imagine that a mixture of these two gas species would produce a very complicated superposition of the two spectra in Fig. 7, whereas the resulting $\mathrm{THz}$ waveform would simply contain two echoes, rather than one. The nature of these time-domain signals affords a great deal of flexibility in a gas sensing system, both with regard to the number and types of gases to be detected.

\section{Linear predictive coding}

The utility of techniques such as those outlined above will depend on the accuracy with which spectroscopic information can be extracted from the time-domain waveform, relative to the differences between the signatures of different gases. This will depend on the effectiveness of the signal processing algorithms used for data analysis. Indeed, the ultimate sensitivity limits of this technique will likely be determined by the degree to which one can extract the signature of a particular gas from a waveform containing a combination of complex timevarying signals. Because the necessary algorithms are under current development, it is premature to estimate sensitivity limits based on the data obtained to date. However, a discussion of the most recent results serves to illustrate the potential of this technology. These results, which rely on a well-known time-domain waveform analysis technique known as linear predictive coding (LPC), are described here.
The value of linear prediction in descriptions of timedomain digital signals which exhibit narrow spectral features is recognized in the field of automated speech recognition [40]. The analysis of a digitally sampled $\mathrm{THz}$ waveform $E(t)$ begins with the assumption that the action of the gas or gas mixture can be modeled as a time-invariant linear filter, of the form

$E(t)=\sum_{k=0}^{M} a_{k} E(t-k \Delta)$.

Here, $\Delta$ is the sampling interval. The coefficients $a_{k}$ are the parameters of the linear prediction, and thus contain the information on the spectral content of the waveform. This can be seen by taking the discrete-time Fourier-transform of (1), to find the frequency response of the transfer function corresponding to this time-domain filter:

$H(f)=\left(1-\sum_{k=0}^{M} a_{k} \exp [-\mathrm{i} 2 \pi k f \Delta]\right)^{-1}$

Equation (2) represents an infinite impulse response (IIR) filter; the coefficients $a_{k}$ determine the poles of the filter function. These coefficients are determined directly from the signal waveforms using a correlation analysis. Here, one minimizes the squared sum of the difference between the measured waveform and that generated by the filtering process. This analysis yields a system of linear equations that relate the LPC coefficients to values of the autocorrelations of the sampled waveform. These equations can be solved using fast matrix inversion techniques such as the Levinson algorithm [40], providing a fast and simple means for generating an $M$-dimensional vector $\boldsymbol{a}=\left\{a_{1}, a_{2}, \ldots a_{M}\right\}$ that can be used to parameterize any measured waveform. It should be noted that, because these coefficients depend only on the autocorrelation of the time-domain waveform, all information involving the relative phases of the oscillatory signals is lost. This could pose a substantial problem in certain cases, as discussed below.

There are two important advantages of using LPC for the parameterization of $\mathrm{THz}$ waveforms for gas recognition. First, an expansion in a series of poles provides a parsimonious representation of a power spectrum with sharp spectral features. LPC methods are particularly well suited for extracting the frequencies and amplitudes of a collection of slowly decaying sinusoids from a superposed waveform. This is in contrast to a Fourier expansion, which can require a large number of coefficients to represent narrow spectral lines. Second, the number of coefficients used to represent each waveform, $M$, is the same for all gases, regardless of the number of absorption resonances. This greatly facilitates the comparison of different waveforms as it permits simple geometric representations. An equivalent treatment using Fourier analysis would necessitate some form of template matching, and would be more cumbersome to implement. On the other hand, the optimal choice of the order parameter $M$ can be problematic, depending on the selection of gases to be sensed. This difficulty is discussed further below.

The data compression inherent in the LPC analysis can be illustrated by comparing the spectral estimation of the filter function of a given sample, using both an LPC analysis 


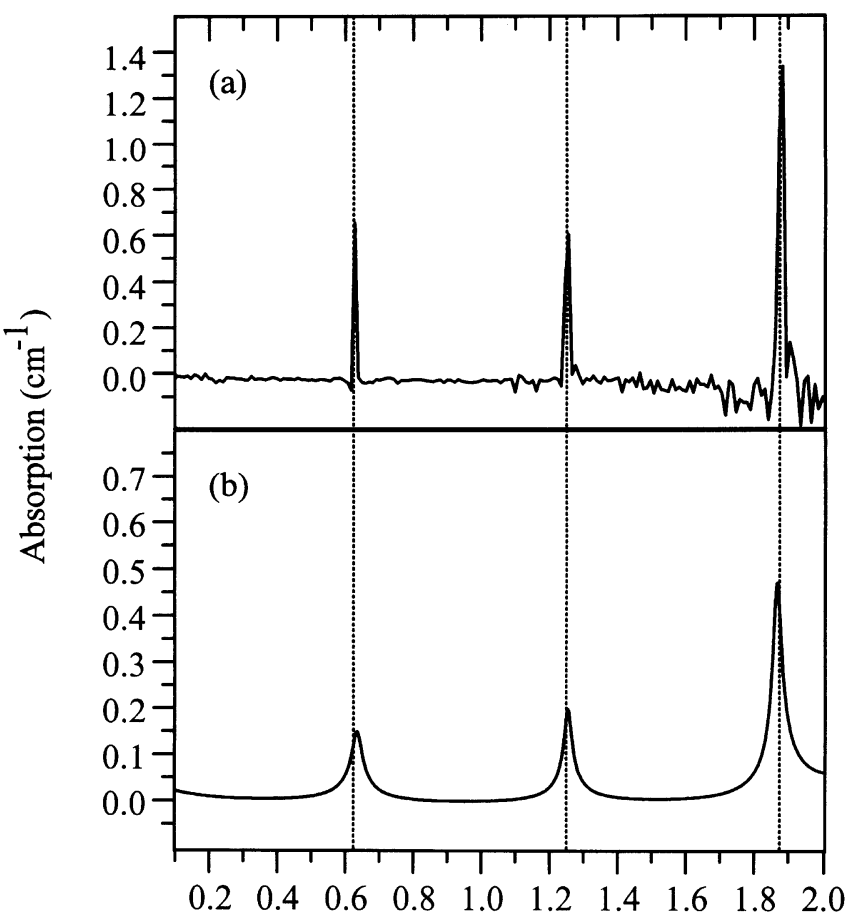

Frequency (THz)

Fig. 8a,b. A comparison of the absorption spectra of $\mathrm{HCl}$ derived from the data by two different methods. a Squared amplitude of the ratio of the Fourier transforms of the signal and reference. b Power spectrum of the filter function obtained from an LPC analysis of the time-domain data. The dashed lines indicate the theoretically predicted positions of the three lines, at $0.626 \mathrm{THz}, 1.251 \mathrm{THz}$, and $1.876 \mathrm{THz}$, from [35]

and a Fourier analysis. An example is shown in Fig. 8. Here, the absorption spectrum of $\mathrm{HCl}$ gas derived from measured time-domain data using Fourier analysis is compared to that derived using LPC analysis. The upper curve (a) is the first 182 points of the spectrum obtained from the squared amplitude of the ratio of the Fourier transforms of the signal and reference waveforms. The lower curve (b) shows the power spectrum of the all-pole filter $|H(f)|^{2}$, using the $M=16$ coefficients derived from the time-domain analysis. Although the spectral resolution is somewhat degraded in (b) relative to (a), the positions and relative amplitudes of the three resonances are quite accurate. This is accomplished with a data compression of more than one order of magnitude. It should be emphasized that power spectra such as the one displayed in Fig. 8b are not used for recognition purposes, but only as a confidence measure for the LPC analysis.

\section{Single-species and mixture analysis}

In order to operate a gas sensing system based on these concepts, experiments are performed on each gas of interest, at known concentrations and path lengths. The resulting $\mathrm{THz}$ waveforms are pre-processed, as described below, then parameterized using an LPC algorithm. The resulting $M$ vectors are stored in a codebook. The final codebook consists of a set of $p$ linearly independent vectors $\left\{\boldsymbol{a}_{i}\right\}$, one for each gas of interest, that span a $p$-dimensional subspace of the $M$-dimensional vector space. An unknown gas sam- ple can then be characterized by measuring the $\mathrm{THz}$ pulse that has passed through it, parameterizing this waveform by its own $M$ vector, and comparing this unknown to each of the vectors representing known gases in the codebook. One possible method of comparison between two such vectors is simply the Euclidean distance between the two. Then, a single-species recognition consists of calculating this distance between the unknown waveform and each of the known waveforms, and using the minimum distance to identify the gas. With no additional processing beyond that mentioned above, this procedure is approximately equivalent in accuracy to a Fourier-based peak height analysis for single-species recognition [30], although of course easier to implement in a real-time automated system.

The analysis of gas mixtures relies on the fact that the LPC vector which characterizes a mixture is simply the vector sum of the codebook vectors representing the individual gases in the mixture, weighted by the partial pressures of each. Hence, a coordinate representation of the vector representing the unknown sample in the $\left\{\boldsymbol{a}_{i}\right\}$ basis may be regarded as a measure of the mole fractions of the species present in the mixture. In practice, an orthogonal basis $\left\{\boldsymbol{b}_{i}\right\}$ is constructed, and used to calculate the projections of the vector representing the unknown gas mixture. These are then related to the projections in the $\left\{\boldsymbol{a}_{i}\right\}$ basis by a linear transformation.

To demonstrate the use of THz-TDS for mixture analysis, binary mixtures of $\mathrm{NH}_{3}$ and $\mathrm{H}_{2} \mathrm{O}$ were studied. These particular gases are not of great interest for far-infrared gas sensing, as they can both be easily detected using vibrational spectroscopy. Rather, they are interesting because resonances at $0.572 \mathrm{THz}\left(\mathrm{NH}_{3}\right)$ and $0.557 \mathrm{THz}\left(\mathrm{H}_{2} \mathrm{O}\right)$, at $1.168 \mathrm{THz}\left(\mathrm{NH}_{3}\right)$ and $1.163 \mathrm{THz}\left(\mathrm{H}_{2} \mathrm{O}\right)$, and again at $1.763 \mathrm{THz}\left(\mathrm{NH}_{3}\right)$ and $1.800 \mathrm{THz}\left(\mathrm{H}_{2} \mathrm{O}\right)$ are nearly coincident within the spectral resolution of the THz-TDS system $[32,35]$. This is shown in Fig. 9, where the experimentally measured intensity absorption spectrum of water vapor is illustrated, along with a stick spectrum showing the five transitions of the ammonia molecule. Because of the overlap between many of the spec-

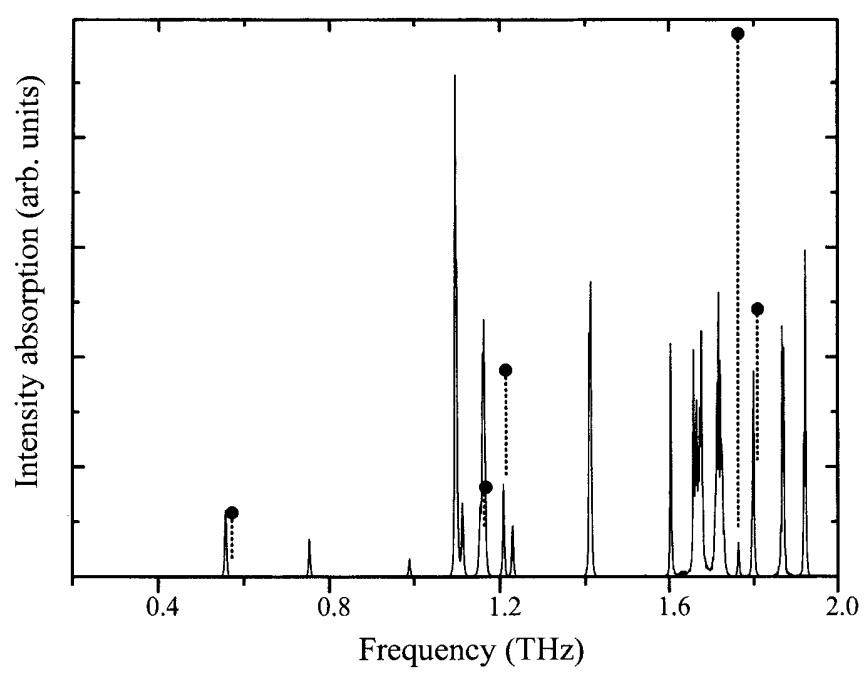

Fig. 9. Intensity absorption spectrum of water vapor from 0.2 to $2.0 \mathrm{THz}$, with a spectral resolution of $\approx 2 \mathrm{GHz}$. Dots and dashed drop lines indicate the positions and intensities of the five rotational transitions of ammonia which fall within this spectral window. Ammonia data are theoretical predictions, obtained from [35] 


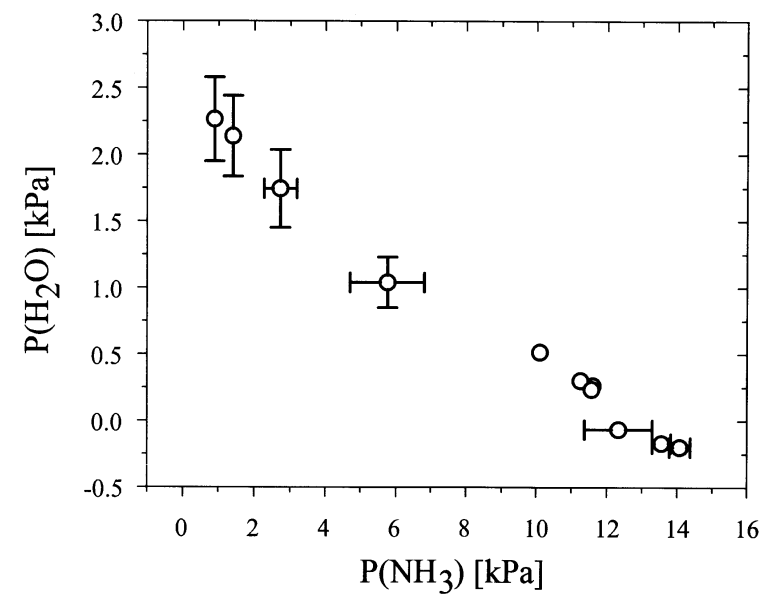

Fig. 10. Results of chemical recognition tests on binary gas mixtures. The data represent a series of measurements on successive gas mixtures, showing the estimated partial pressures of the two gases. Error bars are estimated as described in the text, and are only shown when they are larger than the data point

tral lines of $\mathrm{NH}_{3}$ with $\mathrm{H}_{2} \mathrm{O}$ lines, this represents a challenging example for mixture analysis.

The results of the experiment are summarized in Fig. 10. Initially, the gas cell is filled with pure $\mathrm{NH}_{3}$ gas at a pressure of $12 \mathrm{kPa}$. Successively, this gas is exchanged with water vapor in portions of a few percent per volume. For each mixture, a waveform is recorded and analyzed. From the results of the LPC analysis and the geometric interpretation of the resulting vector, mole fractions of the two species are determined. These are converted into partial pressures by scaling to the pressures used in the measurements of the corresponding codebook vectors. Nonvanishing projections onto vectors corresponding to species in the codebook that were not

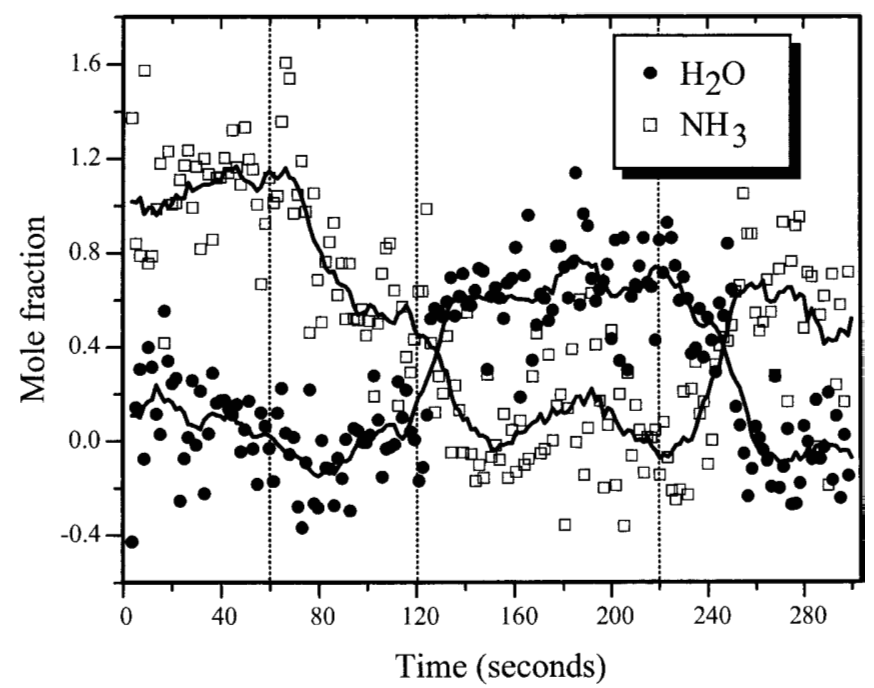

Fig. 11. Real-time gas mixture analysis using the LPC analysis of $\mathrm{THz}$ waveforms. Each pair of data points (one for each gas) represents $2 \mathrm{~s}$ of signal averaging. The solid lines represent a 20 -s running average. The first vertical dashed line indicates the time at which the slow removal of ammonia was begun, the second shows the time at which water vapor was introduced into the cell, and the third shows the initiation of an exchange of the two gases present in the gas cell $\left(\mathrm{HCl}\right.$ and $\left.\mathrm{CH}_{3} \mathrm{CN}\right)$ are attributed to experimental uncertainty, and used to estimate error bars.

The speed of the processing is demonstrated in Fig. 11, which shows the results of mixture analysis implemented in a real-time sensing mode. Here, the gas cell is filled initially with pure $\mathrm{NH}_{3}$. At $t=0$, the system begins to analyze the contents of the cell using the methods outlined above. A pair of data points (one for each gas) is plotted every two seconds, representing the results of an LPC analysis of the average of forty $\mathrm{THz}$ waveforms. The vertical dashed lines in the figure indicate the times at which changes in the contents of the cell were initiated, as described in the figure caption. As in Fig. 10, the system is able to track the changes in the mixture composition, from a pure $\mathrm{NH}_{3}$ mixture to one dominated by $\mathrm{H}_{2} \mathrm{O}$, as well as detect simultaneous changes in the partial pressures of both gases.

\section{Discussion}

\subsection{Waveform pre-processing}

In the data presented here, the waveforms have been preprocessed prior to the LPC analysis. This procedure consists of a time-domain windowing to remove the initial transient from the data. Failure to do so can skew the results of predictions, because of the large difference in amplitude between this feature and the subsequent oscillations. Additional filtering serves to remove small low-frequency components from the waveforms, which further stabilizes the linear prediction. Here, we discuss a potentially valuable enhancement to this pre-processing procedure.

In order to accurately extract information from a measured waveform, it is necessary to distinguish between those features which result from the interaction of the radiation with the sample under study and those which are intrinsic to the $\mathrm{THz}$ system, i.e., the instrument response function. The preliminary results involving gas sensing can be substantially improved by appropriate deconvolution of these features, prior to the analysis with the LPC algorithm. The need for such pre-processing is illustrated in Fig. 12, which shows the $\mathrm{THz}$ waveform transmitted through a cell, both (a) without and (b) with a sample gas $(\mathrm{HCl})$ present. Curve (b) (signal) displays the distortions imposed on the waveform, in the time domain, by the absorption in the gas. Note that the "ringing" which constitutes the signature of the gas closely resembles the features which follow the main pulse in curve (a) (reference). These features (largest ones indicated by arrows) are a result of either electrical or optical reflections, due to impedance mismatches in the emitter antennas, multiple reflections in the cell windows, or similar effects. These are characteristic of the $\mathrm{THz}$ system, and not of the action of the gas, and they therefore appear identically in both waveforms. This close resemblance between features of the reference waveform and the signatures of the gas can limit the effectiveness of any estimation algorithm, including LPC. Evidently, a robust means for deconvolving this known system response function, including both the initial peak and the subsidiary reflections, will improve the performance of the system.

One obvious solution is to perform a deconvolution of these features by means of a ratio in the Fourier domain. 


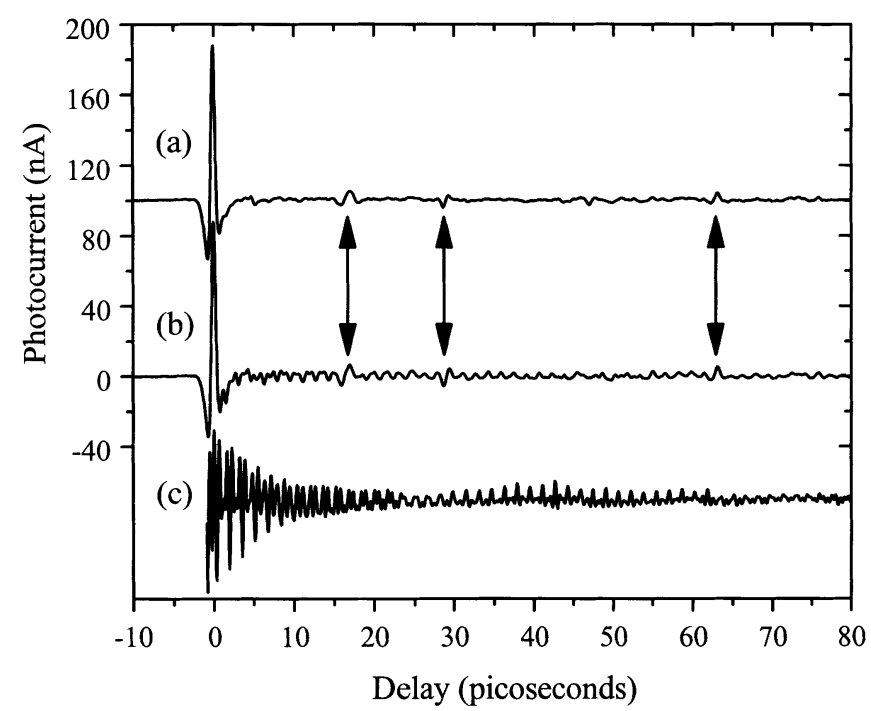

Fig. 12. Reference (a) and signal waveforms (b), showing the effects of $\mathrm{HCl}$ vapor. Arrows indicate several features in these waveforms which are characteristic of the system response, arising from electrical and optical reflections, and which are not a result of the action of the gas. Curve (c) illustrates the deconvolution of the system response (a) from the signal (b), leaving only the slowly damping sinusoidal functions which arise from the three rotational absorption lines (see Fig. 8). The three curves have been vertically offset for clarity

However, it is desirable to design a linear filter that simultaneously deconvolves the unwanted impulse response and discriminates against measurement noise. A Wiener deconvolution filter balances deconvolution and noise filtering. The transfer function of such a filter is given by

$G(f)=\frac{I^{*}(f) P(f)}{|I(f)|^{2} P(f)+\sigma_{n}^{2}}$,

where $I(f)$ is the spectrum of the impulse response, $P(f)$ is the power spectrum of the input, and $\sigma_{n}^{2}$ is the variance of the measurement noise, assumed here to be white noise [41]. The power spectrum of the input is not directly measured here, but a reasonable approximation can be obtained by simply inserting the known high-frequency cutoff of the spectrum, $\approx 2.1 \mathrm{THz}$, in this example. The result of this simultaneous denoising and deconvolution is shown in Fig. 12c. Here, all of the features in the time-domain waveform which appear in the reference have been removed, leaving only the oscillatory features induced by the gas.

\subsection{LPC analysis}

Although LPC analysis is one of the most widely used spectral estimation techniques, it is by no means the only procedure for rapid parameterization of time-varying signals. The preliminary results presented here represent a proof of principle, but are certainly not optimized, even for the gases used in the examples above. Indeed, several aspects of the results presented in Figs. 10 and 11 can be attributed to either deficiencies in the implementation of the LPC code in our experiment, or the inappropriateness of an LPC analysis in parameterization of the data. Here, these problems are discussed, and several possible solutions proposed.
In Fig. 10, when the mixture is mostly $\mathrm{NH}_{3}$ (lower right), the prediction yields a slightly negative pressure for $\mathrm{H}_{2} \mathrm{O}$, while overestimating the $\mathrm{NH}_{3}$ pressure. Similar effects are observed in Fig. 11, where a substantial scatter is observed about the mean pressure values for both gases. These effects result in part from insufficient statistics for the coded vectors. The use of well-known clustering techniques [42] in the parameterization of the codebook vectors should improve this performance. Also, a more elaborate pre-processing of the measured $\mathrm{THz}$ waveforms, such as that described above, should enhance the stability of the subsequent analysis and improve the accuracy of the results. Finally, although it is intuitively satisfying to parameterize the degree of similarity between the spectra of two estimated waveforms by the Euclidean distance between the two vectors of LPC coefficients, it is by no means obvious that this is reliable, or even unique for a given pair of gases. In fact, a more appropriate metric, commonly used in speech recognition applications, is the Itakura-Saito distortion measure, given by

$$
d_{\mathrm{IS}}=\int_{-1 / 2 \Delta}^{1 / 2 \Delta}[\exp \{V(f)\}-V(f)-1] \mathrm{d} f
$$

As above, $\Delta^{-1}$ is the sampling frequency. $V(f)$ is the $\log$ spectral difference between the two waveforms under consideration, $S_{1}(f)$ and $S_{2}(f)$ :

$V(\omega)=\log S_{2}(f)-\log S_{1}(f)$.

Minimizing the Itakura-Saito distance $d_{\mathrm{IS}}$ has been shown to be equivalent to a minimization of the energy difference between two waveforms [42]. This distance can be re-expressed in terms of autocorrelations of the temporal waveform, for compatibility with a time-domain analysis.

Beyond the question of distance estimation, a number of other difficulties can be expected to arise with any LPC-based description. The most obvious involves the choice of the order parameter $M$, the number of coefficients used to describe each waveform. Obviously, larger values of $M$ lead to more accurate estimations of the spectral information contained in a waveform. However, if an all-pole filter of order $M$ is used for estimation of a signal containing fewer than $M$ peaks, then the description is not necessarily unique. This will inevitably lead to errors in gas analysis, as two entirely different sets of coefficients can describe the same gas. Thus, choosing an optimal number of coefficients is not a trivial task. Depending on the particular set of gases of interest, there may be no value of $M$ that permits sufficient discrimination between two similar gases but still provides a unique description of each gas. Clustering techniques can address this difficulty in some cases, but it may be necessary to explore other algorithmic approaches to characterization of waveforms. Alternative methods for waveform decomposition, including Prony's method and eigenspace methods [43], are currently under investigation.

A further problem arises from the loss of relative phase information mentioned above. Consider the two examples depicted in Fig. 6. Gases such as these, where the regularly spaced rotational manifold gives rise to echoes in the time domain, are most conveniently characterized by the delay 
between the initial transient and the first reflection. As described above, this delay is inversely proportional to the rotational constant of the molecule. If these waveforms are pre-processed by removing the initial transient (see Fig. 12c), then the only remaining vestige of the magnitude of the rotational constant lies in the temporal delay at which the echoes arise. This phase information is lost when an autocorrelation is performed. Thus, the LPC cannot readily distinguish between these two gases. In fact, because of the large number of sinusoids (i.e., the large number of absorption lines in the rotational manifold) present in these particular examples, these represent gases for which an LPC analysis is least appropriate, and thus most likely to fail or become unstable. A wavelet description is more well suited for analysis of gases such as these, for which the time-domain signature is highly localized.

\section{Wavelet processing of $\mathrm{THz}$ waveforms}

Since $\mathrm{THz}$ pulses are localized in both time and frequency, they are naturally suited to signal processing methods based on wavelets. The wavelet transform performs a "local Fourier analysis" by analyzing and representing signals in terms of shifted and dilated versions of time-localized, oscillating functions [44]. Since the elements of a wavelet basis can be designed to closely resemble the underlying waveforms in the $\mathrm{THz}$ system, wavelet-based signal processing algorithms will outperform more traditional techniques. Indeed, it has been shown that noise removal, compression, and signal recovery methods based on wavelet coefficient shrinkage or wavelet series truncation enjoy excellent asymptotic performance and moreover, do not introduce excessive artifacts in the signal reconstruction [45]. Thus wavelets appear to be a natural tool for addressing the processing challenges presented by the THz-TDS system.

Measurement noise is an issue which will inevitably limit the performance of the sensing system, particularly as the speed of waveform acquisition is increased. Removing noise from a measured waveform prior to processing will be an important aspect of the pre-processing procedure. Waveletbased denoising will be far superior to the more familiar Fourier-based techniques $[45,46]$, particularly when the raw data resembles the waveforms of Fig. 6. Because the elements of a wavelet basis can be tailored to closely resemble these THz-TDS signals, fewer coefficients are required to represent the signals in a wavelet basis than in a Fourier expansion. This is illustrated in Fig. 13, using the waveform of Fig. 14a as an example. Here, (a) and (b) depict the representations of this data in the Fourier and wavelet bases, respectively. Because the wavelet transformation is a mixed time-frequency representation, the wavelet decomposition of a temporal waveform is displayed in a two-dimensional format, as shown. This display (b) shows that the wavelet representation requires a small number of coefficients of significant amplitude, mostly localized along the time axis at the two positions where the waveform is large. In (c), the 1024 coefficients in these two expansions are sorted in descending order, and displayed on a logarithmic scale. The Fourier coefficients decay more slowly, confirming that more large coefficients are required for an accurate representation of the signal. Also, the noise floor at $\approx 10^{-3}$ of the peak sig-

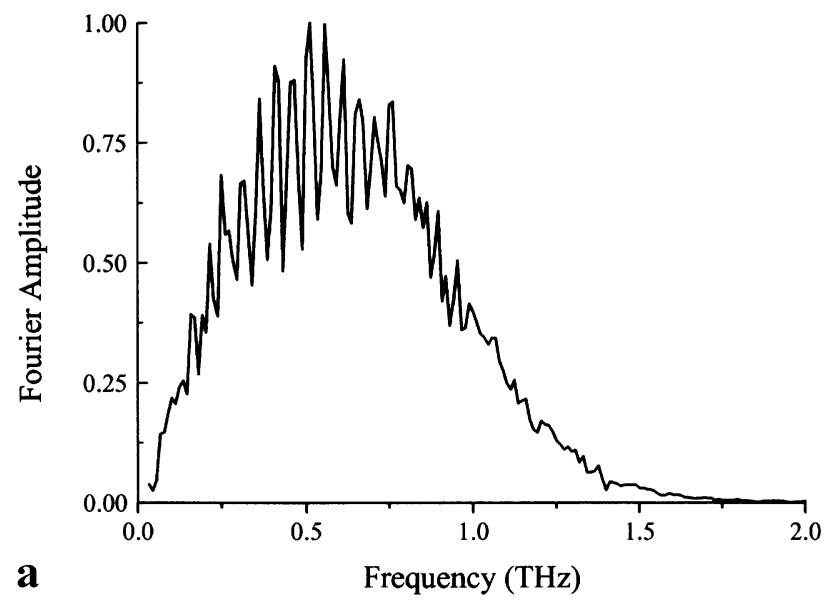

b
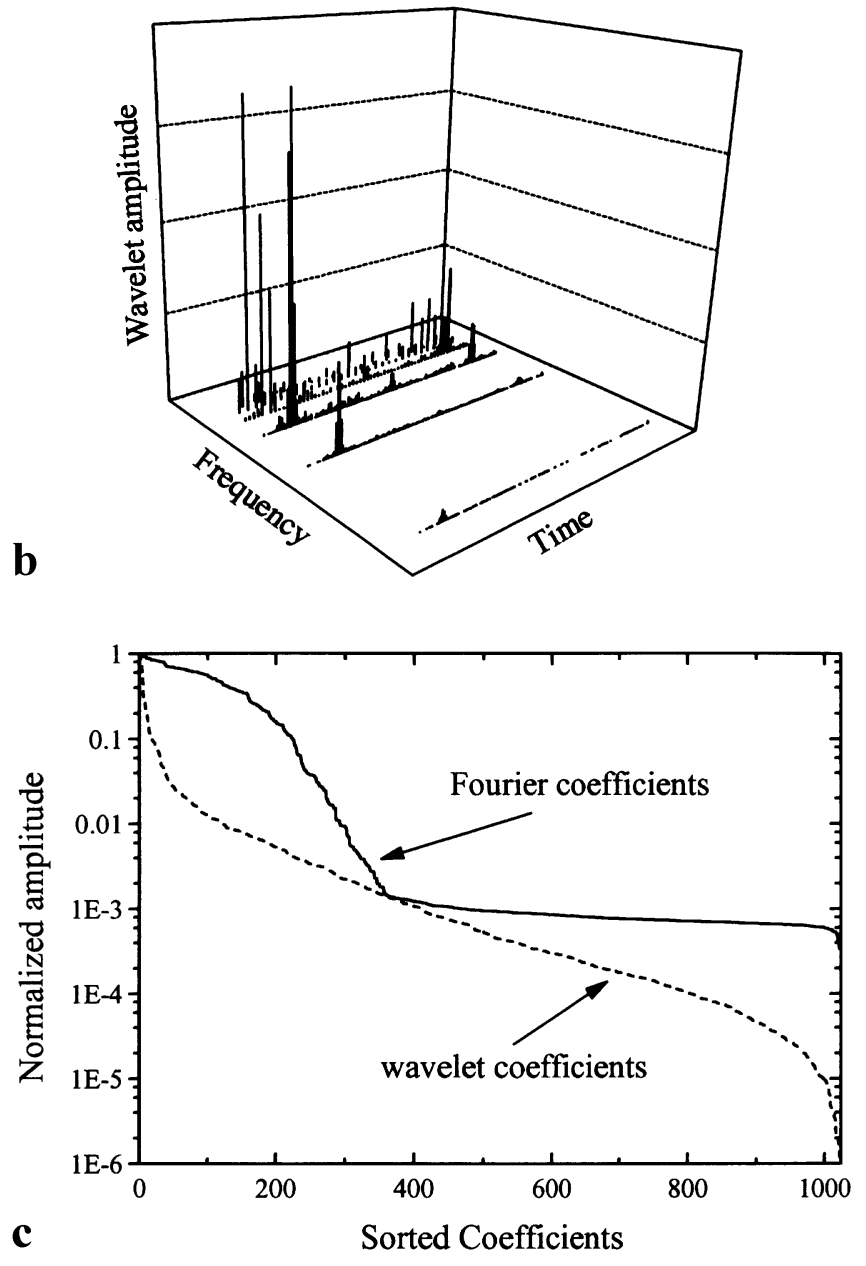

Fig. 13a-c. $\mathrm{THz}$ waveform of Fig. 14a represented in Fourier (a) and wavelet (b) bases. In (c), the coefficients of these two expansions are sorted in descending order, to illustrate the extremely rapid decay of the wavelet coefficients relative to the Fourier representation. Wavelets permit an extremely compact representation of the $\mathrm{THz}$ waveforms, which facilitates threshold denoising

nal is evident, where the curve exhibits a "knee" followed by a noise-limited plateau. A simple Fourier-based denoising would consist of truncation of these coefficients at this point, thus reducing the number of non-zero coefficients from 1024 to $\approx 360$. The wavelet coefficients decay far more rapidly, 


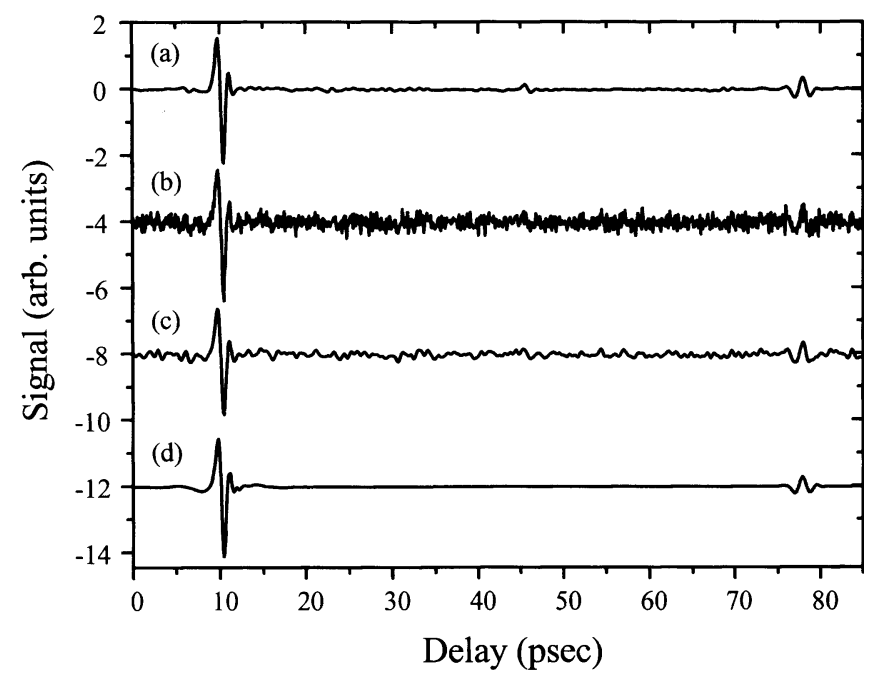

Fig. 14. A comparison of the wavelet and Fourier-based denoising schemes described in the text. (a) A sample unprocessed waveform, acquired under similar conditions to Fig. 6b. (b) The same waveform, with Gaussian white noise added to artificially simulate noisy data. The rms noise amplitude is approximately $10 \%$ of the peak-to-peak signal amplitude. (c) The noisy waveform (curve (b)), denoised using a parabolic filter in the Fourier domain, with a cutoff frequency of $1.94 \mathrm{THz}$, and (d) denoised using wavelet soft thresholding. Waveforms vertically offset for clarity

thus permitting a truncation much sooner. This enables a far more efficient denoising strategy, as well as substantial signal compression. Figure 14 shows a comparison of denoising of a THz waveform using these two methods. Here, a THz waveform is artificially supplemented with white Gaussian noise, and subsequently processed using both a parabolic Fourier filter and a soft threshold in the wavelet domain. The wavelet denoising is evidently far superior for this type of noise. The development of a wavelet-based denoising strategy for $1 / f$ noise, of the type expected in these measurements, is a topic of current research.

\section{Conclusion}

The use of terahertz time-domain spectroscopy for gas sensing based on far-infrared identification of fingerprint spectral features has been demonstrated. Both single-species recognition and quantitative gas mixture analysis are possible, using the recently developed real-time waveform acquisition capability of THz-TDS in combination with powerful signal processing algorithms originally written for speech recognition purposes. The signals measured in THz-TDS can in general consist of superpositions of signatures from several different gases, as well as a non-trivial instrument response which can mimic or distort the response due to gases. The extraction of the desired information from such signals using conventional Fourier methods quite rapidly reaches the limits of its utility, and one is naturally led to more sophisticated signal processing approaches. The ultimate sensitivity limits of this device for a given gas will depend largely on the effectiveness of these procedures. Fortuitously, the THz-TDS waveforms belong to a large class of signals for which wavelet processing is extremely effective. We anticipate that the incorporation of wavelet-based algorithms and improved recognition proced- ures will greatly enhance both the sensitivity and accuracy of the system. Because certain gas species are more appropriate than others for a wavelet-based analysis, the optimal signal processing scheme will depend to a degree on the particular gases to be sensed in any given situation. Thus, pattern matching for gas identification will probably rely on some form of template training. The training problem is a well-known challenge in speech recognition [42], and we expect that effective algorithmic approaches can again be borrowed from this advanced field for gas sensing purposes. With the concurrent development efforts aimed at producing a commercially viable portable $\mathrm{THz}$ spectrometer, the prospects for $\mathrm{THz}$ gas sensing are quite bright.

Acknowledgements. We thank Y. LeCun for helpful discussions. The use of the FTIR spectrometer in the group of V. Colvin at Rice University is gratefully acknowledged.

\section{References}

1. J.A. O’Neill, M.L. Passow, T.J. Cotler: J. Vac. Sci. Technol. A 12, 839 (1994)

2. K.P. Petrov, S. Waltman, U. Simon, R.F. Curl, F.K. Tittel, E.J. Dlugokencky: Appl. Phys. B 61, 553 (1995)

3. P.R. Smith, D.H. Auston, M.C. Nuss: IEEE J. Quantum Electron. QE24, 255 (1988)

4. B.B. Hu, X.-C. Zhang, D.H. Auston: Phys. Rev. Lett. 67, 2709 (1991)

5. L. Xu, X.-C. Zhang, D.H. Auston: Appl. Phys. Lett. 61, 1784 (1992)

6. M.C. Nuss, P.C.M. Planken, I. Brener, H.G. Roskos, M.S.C. Luo, S.L. Chuang: Appl. Phys. B 58, 249 (1994)

7. K. Kawase, M. Sato, T. Taniuchi, H. Ito: Appl. Phys. Lett. 68, 2483 (1996)

8. S. Verghese, K.A. McIntosh, E.R. Brown: IEEE Trans. Microwave Th. Tech. 45, 1301 (1997)

9. Y.R. Shen: Prog. Quantum Electron. 4, 207 (1976)

10. S. Tsuda, W.H. Knox, S.T. Cundiff: Appl. Phys. Lett. 69, 1538 (1996)

11. M.E. Fermann, D. Harter, J.D. Minelly, G.G. Vienne: Opt. Lett. 21, 967 (1996)

12. M. van Exter, D.R. Grischkowsky: IEEE Trans. Microwave Th. Tech. 38, $1684(1990)$

13. P. Uhd Jepsen, R.H. Jacobsen, S.R. Keiding: J. Opt. Soc. Am. B 13, 2424 (1996)

14. D.M. Mittleman, R.H. Jacobsen, M.C. Nuss: IEEE J. Sel. Top. Quantum Electron. 2, 679 (1996)

15. R.H.M. Groeneveld, D. Grischkowsky: J. Opt. Soc. Am. B 11, 2502 (1994)

16. M.C. Nuss, D.H. Auston, F. Capasso: Phys. Rev. Lett. 58, 2355 (1987)

17. D.M. Mittleman, J. Cunningham, M.C. Nuss, M. Geva: Appl. Phys. Lett. 71, 16 (1997)

18. G. Haran, W.-D. Sun, K. Wynne, R.M. Hochstrasser: Chem. Phys. Lett. 274, 365 (1997)

19. B.B. Hu, M.C. Nuss: Opt. Lett. 20, 1716 (1995)

20. Q. Wu, F.G. Sun, P. Campbell, X.-C. Zhang: Appl. Phys. Lett. 68, 3224 (1996)

21. D.M. Mittleman, S. Hunsche, L. Boivin, M.C. Nuss: Opt. Lett. 22, 904 (1997)

22. P.R. Smith, D.H. Auston, M.C. Nuss: IEEE J. Quantum Electron. QE24, 255 (1988)

23. Ch. Fattinger, D. Grischkowsky: Appl. Phys. Lett. 54, 490 (1989)

24. F.E. Doany, D. Grischkowsky, C.-C. Chi: Appl. Phys. Lett. 50, 460 (1987)

25. A. Nahata, D.H. Auston, T.F. Heinz, C. Wu: Appl. Phys. Lett. 68, 150 (1996)

26. Q. Wu, X.-C. Zhang: Appl. Phys. Lett. 71, 1285 (1997)

27. I. Brener, Q. Wu, Y. Cai, X.-C. Zhang, J. Lopata, J. Wynn, L. Pfeiffer, J.B. Stark, J. Federici: In Conference on Lasers and Electro-Optics, 11, 1997 OSA Technical Digest Series (Optical Society of America, Washington, DC 1997) pp. 136-7 
28. R.A. Cheville, D. Grischkowsky: Opt. Lett. 20, 1646 (1995)

29. A.F. Krupnov: Spectrochim. Acta, Part A 52, 967 (1996) and references therein

30. R.H. Jacobsen, D.M. Mittleman, M.C. Nuss: Opt. Lett. 21， 2011 (1996)

31. N. Gopalsami, S. Bakhtiari, A.C. Raptis, S.L. Dieckman, F. De Lucia: IEEE Trans. Instrum. Meas. 45, 225 (1996)

32. M. van Exter, Ch. Fattinger, D. Grischkowsky: Opt. Lett. 14, 1128 (1989)

33. H. Harde, N. Katzenellenbogen, D. Grischkowsky: J. Opt. Soc. Am. B 11, 1018 (1994)

34. H. Harde, D. Grischkowsky: J. Opt. Soc. Am. B 8, 1642 (1991)

35. H.M. Pickett, R.L. Poynter, E.A. Cohen: Submillimeter, Millimeter, and Microwave Spectral Line Catalog, accessible on the World Wide Web (http://spec.jpl.nasa.gov) from the Jet Propulsion Laboratory, Pasadena, CA; also see R.L. Poynter, H.M. Pickett: Appl. Opt. 24, 2335 (1985)

36. H. Harde, S.R. Keiding, D. Grischkowsky: Phys. Rev. Lett. 66, 1834 (1991)
37. T.M. Goyette, W. Guo, F.C. De Lucia, J.C. Swartz, H.O. Everitt, B.D. Guenther, E.R. Brown: Appl. Phys. Lett. 67, 3810 (1995)

38. M. Kessler, H. Ring, R. Trambarulo, W. Gordy: Phys. Rev. 79, 54 (1950)

39. F. Cerceau, F. Raulin, R. Courtin, D. Gautier: Icarus 62, 207 (1985)

40. J.G. Proakis, D.G. Manolakis: Digital Signal Processing, Principles, Algorithms, and Applications (Prentice-Hall, Englewood Cliffs, NJ 1996)

41. K.R. Castleman: Digital Image Processing (Prentice-Hall, Englewood Cliffs, NJ 1996)

42. L. Rabiner, B.-H. Juang: In Fundamentals of Speech Recognition, ed. by A.V. Oppenheim (Prentice-Hall, Englewood Cliffs, NJ 1993)

43. S.M. Kay: Modern Spectral Estimation - Theory and Application (Prentice-Hall, Englewood Cliffs, NJ 1988)

44. I. Daubechies: Ten Lectures on Wavelets (Society for Industrial and Applied Mathematics, Philadelphia 1992)

45. D. Donoho: IEEE Trans. Info. Theory 41, 613 (1995)

46. S. Ghael, A.M. Sayeed, R.G. Baraniuk: Proc. SPIE, San Diego, July 1997 